\title{
Field Effect Sensors for PCR Applications
}

\author{
M.Taing, D.Sweatman ${ }^{1}$, \\ CRC for MicroTechnology, Griffith University Microfabrication Laboratory
}

\begin{abstract}
This research focuses on the use of EIS (electrolyte on insulator on Silicon) structure as a detection method for pathogenic DNA binding.

The EIS structure (Electrolyte on Insulator on Silicon) provides a novel, label-free and simple to fabricate way to make a field effect DNA detection sensor. The sensor responds to fluctuating capacitances caused by a depletion layer thickness change at the surface of the silicon substrate and also through DNA adsorption onto the dielectric oxide/amino surface.
\end{abstract}

As DNA molecules diffuse to the sensor surface, they are bound to their complimentary capture probes. The negative charge exhibited by the DNA forces negative charge carriers in the substrate to move away from the surface. This causes an n-type depletion layer substrate to thicken and a p-type to thin.

The depletion layer thickness can be measured by its capacitance using an HP4284a LCR meter. This experiment is conducted using the ConVolt (constant voltage) approach.

Nucleic acids are amplified by an on chip PCR system and then fed into the sensor. The low ionic solution strength will ensure that counter-ions do not affect the sensor measurements. The sensor surface will contain capture probes that will bind to the pathogen. They are held onto the sensor surface by the positively charged amino layer.

\section{Introduction}

Much work on biological sensing has improved dramatically over the decades due to the coupling of microelectronics and biological technologies. In our research, we focus on using techniques (that are normally used in the microelectronics industry for characterizing the quality of devices) to perform the biosensing and detection of biomolecules. Not only this, but one can also improve the sensitivity of the detection by understanding which regime to operate measurements in. This is done primarily by extracting the Capacitance - Voltage curve. Firstly a capacitor is microfabricated in a cleanroom consisting of silicon with a grown oxide layer. The capacitor can then be driven into 3 operating regimes by applying a voltage at the gate of the device - accumulation, depletion and inversion. The gate can be created by depositing a metal on the surface of the oxide or immersing the oxide in a conductive solution (in our case). A bias voltage is applied to the either the metal or solution to drive the device.

To tie this into how the device detects bio-molecules, it is important that we understand that DNA carries a charge. In an experimental or practical situation, DNA will be suspended in the solution that is in contact with the oxide. Over time, DNA will diffuse through the electrolyte towards the surface of the thin oxide which will render it close enough to the silicon sensing surface. The charge carried by the DNA modulates the charged carriers in the silicon and this is how detection takes place. One can measure this electrically by creating two contacts - one in the electrolyte and the other to the silicon to form a simple capacitor device. In the next sections, this paper will explore these concepts further and explain how the experiments were performed and what techniques are used.

\footnotetext{
${ }^{1}$ Primary Correspondant d.sweatman@me.gu.edu.au; phone: 61+(07) 38755050
} 


\subsection{Electrolyte on Insulator on Silicon Structure (EIS)}

In this paper we will discuss micro fabricating the EIS structure to be used as our biological field effect sensor. The structure is shown in figure 1. It consists of a silicon substrate, insulator (Silicon Dioxide) and electrolyte (phosphate buffer solution). The EIS structure is similar to a capacitor in many ways. The silicon can be likened to the bottom plate of a capacitor and the electrolyte (biased by a reference electrode) becomes the top plate. Rather than have electron charges deposited onto a metal contact as in a capacitor, they are deposited by ion diffusion in the electrolyte. These ions are given their potential (energy) via a reference electrode in the electrolyte.

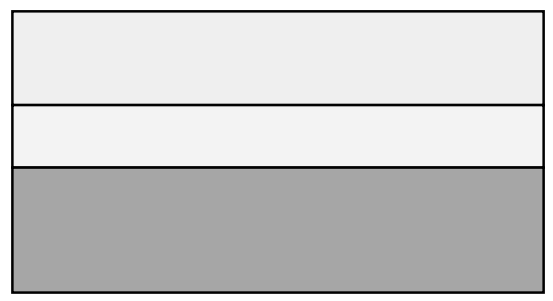

Figure 1. EIS (Electrolyte-Insulator-Silicon)

The charges deposited on the surface of the insulator modulate the charge distribution in the insulator dielectric by creating temporary dipoles. These dipoles influence the surface potential in the silicon beneath, causing charge carriers to move in a doped silicon substrate. The carriers in silicon can either be repelled or attracted to the surface depending on the polarity of surface potential. As majority carriers become sufficiently repelled from the surface, a region is formed containing immobile fixed charges [5]. These charges are immobile because they are now in a state of equilibrium. Their bonding structure is firmly fixed to the crystal lattice since they have lost their "impurity" charge. This region is called the depletion region. This is useful for biological detection because we can modulate the depletion region width by applying a surface charge. Instead of using electrons or solution ions to induce the surface charge, we can use DNA strands. Each polypeptide unit contains a negative charge in its sugary backbone. So if DNA is brought close enough to the silicon surface, it will modulate the depletion region as if it were a capacitor [8]. Therefore, we can tell whether DNA is present or not by measuring the capacitance.

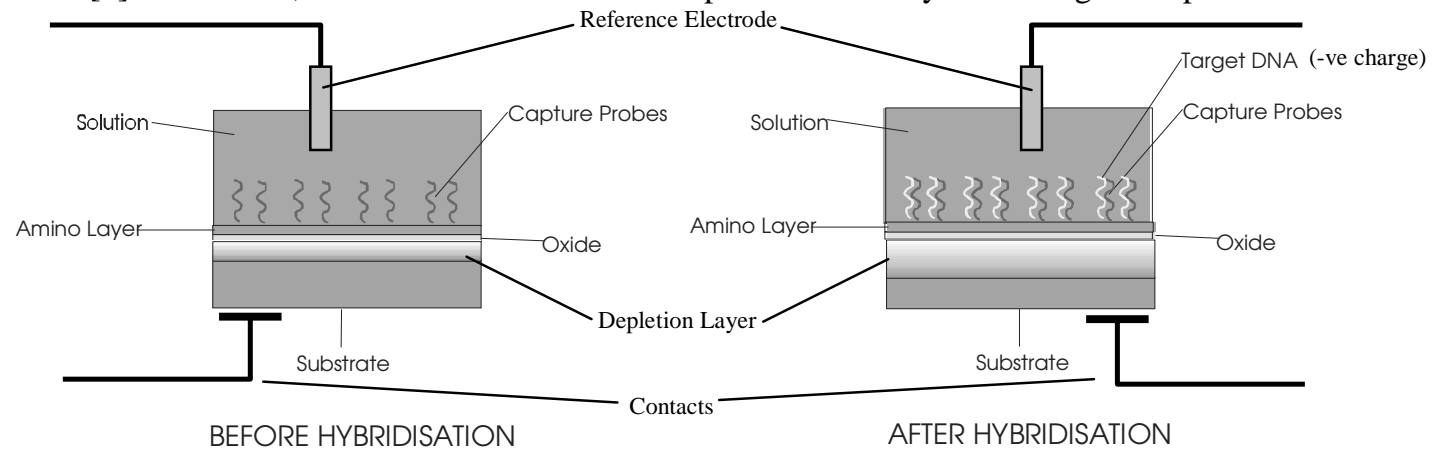

Figure 2. Silicon Depletion Layer Modification due to surface charge

To ensure we attract the DNA of interest to the surface, we must put down a set of capture probes. These are generally short oligonucleotide nucleic acid sequences whose structures are exactly complimentary to the target DNA we wish to sense. However, capture probes don't usually stick very well to silicon dioxide surface. To circumvent this, we need to deposit some sort of adhesion layer between the capture probes and silicon dioxide. A layer consisting of Amino functional end group will serve our purpose here. The adhesion works by simple electrostatic attraction. The first step is to deposit the Amino layer onto the substrate. Before we do that we must prepare the silicon dioxide for optimum adhesion by creating negatively charged surface sites on the oxide. This involves a substitution and oxidation process and is 
described later in this paper [6]. Then we deposit our Amino layer either through spotting or spinning it on silicon. Figure 3 shows the electrostatic attraction that holds the structure together to form the EIS structure. Once that is complete, we are left with a sandwich of layers of the EIS structure.

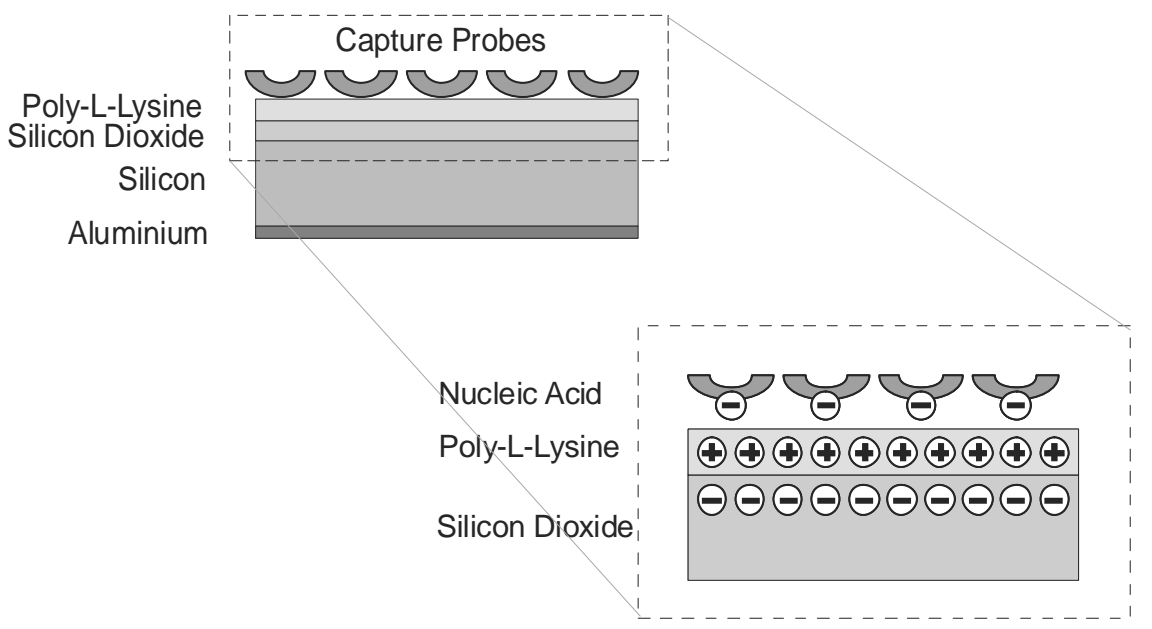

Figure 3. EIS Structure

Now that we have the EIS structure, we need to be able to measure the response to the DNA with the highest sensitivity possible. We desire something that will provide us with a large signal out given a small signal in. Hence we really want the capacitance we are measuring to change by a quantifiable amount given that a minimal amount of DNA adheres to the surface. Here we must introduce the concept of a Capacitance / Voltage (CV) curve. The CV curve is a standard technique used to assess the quality of Metal-Oxide-Silicon capacitors. It gives information such as where the threshold voltage and the flatband voltage are located. However, we are concerned with the flatband voltage only. The threshold voltage is the voltage required to create a channel containing mobile minority carriers all condensed near the surface of the silicon / silicon dioxide interface. This regime is referred to as the inversion regime - which is opposite to the depletion regime which only contains fixed ions. The flatband voltage is the voltage required to ensure no surface potential exists between the silicon and insulator. It is the voltage required to compensate for an excess or deficiency of surface charges (which are causing a surface potential to exist) by removing them [7]. Effectively, it is the voltage required to bring the surface potential back to zero. Hence it removes any surface charges that are present at the silicon surface [7]. If there are no excess charges present at the silicon surface, then there will be no depletion layer. The graph seen in figure 4 is for typical p-type silicon. A negative voltage attracts the majority carrier holes to the surface. The capacitance here is large because the depletion layer has not been formed yet. This is called the accumulation regime. As the voltage increases, more and more majority carriers are being repelled or pushed away from the surface causing the depletion layer to thicken. As the depletion layer thickens, the capacitance decreases. This is represented by the declining slope in the CV curve. Finally, it reaches a point where no matter how much positive potential is applied, the capacitance just doesn't get any smaller. This is known as the inversion regime.

With a CV curve we can tell when surface charges or a surface potential is present. This is seen as a noticeable shift depending on the polarity of the induced potential. A typical shifted CV curve is shown in figure 4 . The dark line shows the original curve and the grey curve shows the shifted curve. If we bias the device at a certain point we can see the amount of change in the capacitance. The amount of shift is directly proportional to surface potential - ie. A larger DNA binding results in a larger shift. Our goal here is to find the optimum bias point so that we get to see a large change in capacitance given a small shift in surface potential. Therefore, to make the sensor as sensitive as possible, we need to locate a spot on the curve with the largest slope. This can be found mathematically by differentiating the curve and looking at the peaks. 


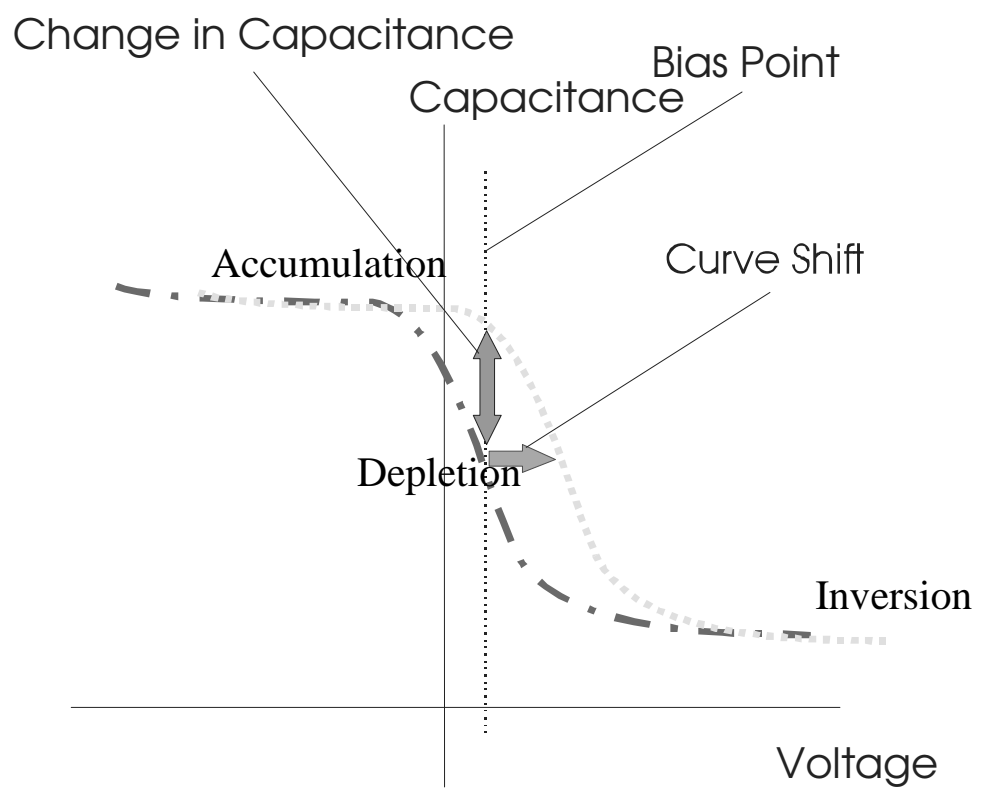

Figure 4. Typical MOS capacitor CV curve (p-type)

This research focuses on using EIS as the generic platform for a biosensor based on the field effect detection principle. The ultimate goal of this research will be to develop a sensor system based on conventional silicon for the detection of food pathogens such as Salmonella, Campylobacter and E.Coli. . Detections are based at the DNA level and not the cellular level. Therefore, specificity is very high. We are striving to increase the detection sensitivity using our differential system. The final product will be seamlessly integrated onto a micro-fluidic biochip incorporating DNA amplification. The field effect sensor has been fabricated into a micro-fluidic channel so it can be incorporated easily and seamlessly onto the biochip system. The biochip system incorporates a system called PCR (Polymerase Chain Reaction) to amplify DNA strands using the traditional thermo-cycling process. The amplified DNA is then pumped through a series of fluidic channels onto our developed sensor platform to perform detection. 


\section{MATERIALS \& METHODS}

\subsection{Sensor}

The EIS structure is a sandwich of layers consisting of silicon, Silicon Dioxide (quartz), biolayer(s) and electrolyte. The substrate layer consists of moderately doped $\left(10^{14}\right) 100$ plane n-type silicon. The substrate is passivated by a layer of $50 \mathrm{~nm}$ thermally grown oxide at $1100^{\circ} \mathrm{C}$ for 30 minutes in an oxygen furnace. The next layer is functionalized with an amino layer to act as the immobilization layer. Finally, specially designed sequences of nucleic acids are to be deposited upon the amino layer which electro-statically adheres in an electrolyte solution. The electrolyte is confined on the sensor by the Teflon stage as shown in figure 5. A home-made $\mathrm{Ag} / \mathrm{AgCl}$ reference electrode provides the stable potential. The home-made reference electrode is made from a Teflon barrel with a ceramic frit on one end. The barrel is filled with a $3 \mathrm{M} \mathrm{KCl}$ gel. A chloride coated silver wire is inserted into the barrel so it makes contact with gel and form the electrical contact between the electrolyte and the silver wire. The home-made reference electrode is compared with a commercial double junction $\mathrm{Ag} / \mathrm{AgCl}$ reference electrode (Ionode IJ14) to ensure its integrity during the experiment. Usually, drifts are less than $10 \mathrm{mV}$ and the impedance is relatively high to prevent polarization of the electrode $(>30 \mathrm{k} \Omega)$. The LCR meter also has an additional $10 \mathrm{M} \Omega$ resistance. The LCR meter (HP4284A) is a computer controlled probe which scans the capacitance whilst sweeping the voltage steps.

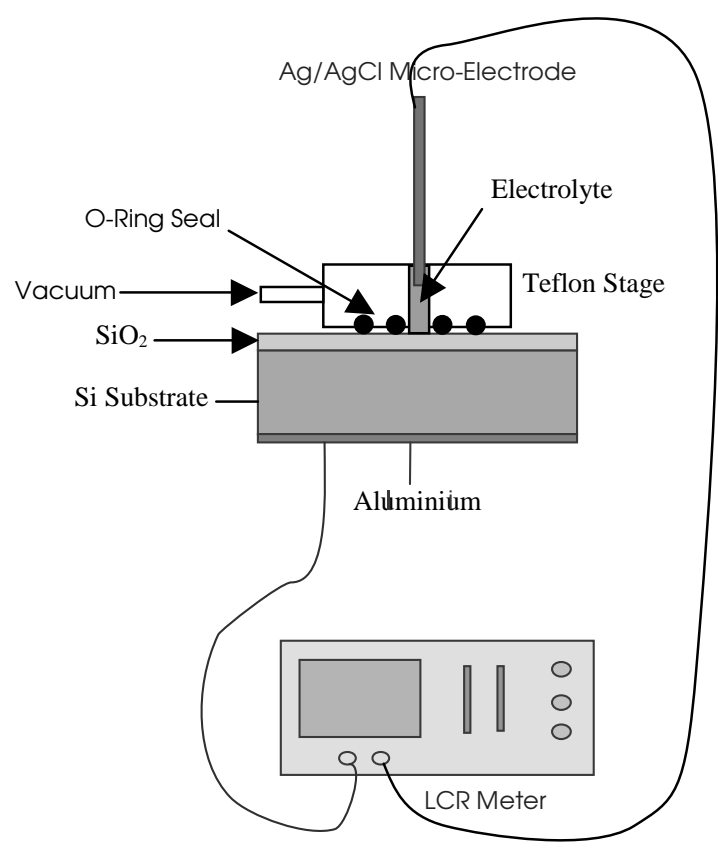

Figure. 5. EIS Structure

The different layers serve a specific purpose. The oxide is there to prevent the solution from leaching onto the silicon substrate and reacting with its surface. The amino layer acts as an immobilization layer by tethering negatively charged capture probes to its binding sites. 


\subsection{Reagents}

The Electrolyte is made up of 1x PBS buffered solution with a measured $\mathrm{pH}$ level of 7.43. The ionic concentration of the solution should be kept at a minimum to reduce the effects of unwanted ions contributing to the surface potential also referred to as background noise [9]. It is known however, that the reduction of ionic concentration reduces the ability of DNA to hybridize. Since each nitrogenous base pair in a DNA strand is equivalent to a negative charge unit, the overall charge of a strand is determined by how long the strand is. Two strands of complementary DNA therefore, tend to have a small electrostatic repelling effect [8]. To compensate for the low ionic concentration and repelling effect, a thin layer of positively charged film is functionalised onto the sensor surface. A suitable compound would contain an amine $\mathrm{R}$ group which easily protonates in a physiological medium. There were many choices when it came to choosing which amine layer to deposit on the oxide. We narrowed these choices down to 2 possible compounds - Poly-L-Lysine or APTES (3-Aminopropyl-triethoxysilane).

Many trials were conducted initially using poly-l-lysine as the adhesion layer, but the results yielded unpredictable behaviour and showed it had an insignificant effect on creating a charged surface.
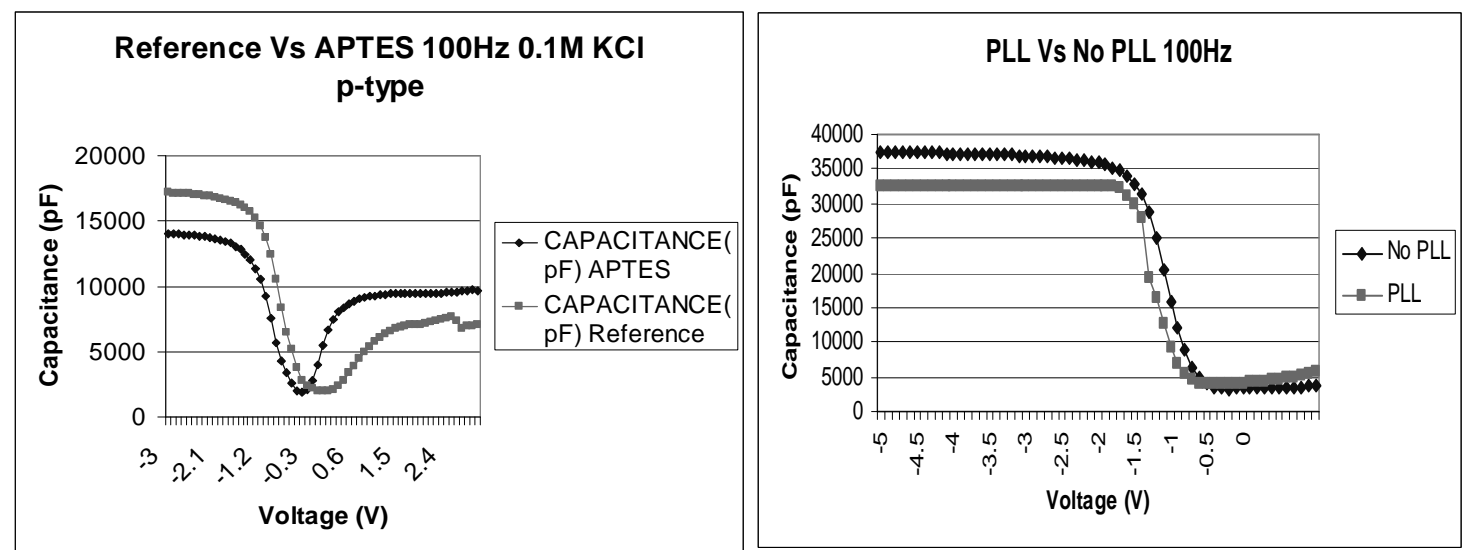

Figure 6. CV curve of coated and non-coated substrates. Left: APTES coated substrate, Right: PolyLysine Coated substrate. Both graphs were sweeped at $100 \mathrm{~Hz}$

Figure 6 shows the comparison when using 2 different types of amino adhesive layers. The left graph shows that APTES creates a much larger shift when compared to using Polylysine - meaning there is a higher density of charged sites on the oxide surface. On most occasions, the shift isn't noticeable when using polylysine. Large concentrations were required to see any charge effect. The concentrations used above were $0.2 \mathrm{mg} / \mathrm{ml}$ (water-base) polylysine and 5\% APTES (5parts APTES, 5parts $\mathrm{diH}_{2} \mathrm{O}$, 90parts $\mathrm{EtOH})$.

Another advantage of using APTES is that it forms a much stronger covalent bond when it is reacted with water on a hydroxyl rich oxide surface. Polylysine on the other hand, attaches to the oxide through a much weaker electrostatic means. To create a rich $\mathrm{OH}$ surface on oxide, we've either treated it in Piranha (1:4 $\mathrm{H}_{2} \mathrm{SO}_{4}: \mathrm{H}_{2} \mathrm{O}_{2}$ ) or gone through a substitution reaction involving the following steps. To achieve this, Silicon Dioxide needs to be thermally grown and treated with boiling Sodium Hydroxide $(\mathrm{NaOH})$ to generate $(\mathrm{Si}-\mathrm{ONa})$. Then immerse into Hydrochloric Acid $(\mathrm{HCl})$ to substitute the Sodium ions with Hydrogen, forming the Silanol group ( $\mathrm{SiOH})[6]$.

Once the oxide is prepped, APTES is deposited by immersing the substrate into a plastic beaker and placed in a shaker for $1 \mathrm{hr}$. The substrate is then washed 3 times in $\operatorname{diH}_{2} \mathrm{O}$ and dried with a steady nitrogen stream. At this stage of the experiment, we have been depositing double stranded DNA rather than placing down capture probes and observing their hybridization with complementary DNA. Currently this is in the process of being done and is a possible discussion for a future paper. 


\section{RESULTS AND DISCUSSION}

\subsection{Measurement Method}

The measurements reported here were taken from the structure shown in figure 5. No amine coating was deposited in these measurements. Here the bare silicon dioxide is directly exposed to the electrolyte. The silicon dioxide becomes the sensitive layer for detecting changes in $\mathrm{pH}$ of a solution.

To optimize the measuring sensitivity, we must find a spot in the C-V curve (capacitance-voltage) where a small change in voltage will result in a large change in capacitance. The change in voltage is caused by a shift in the flatband voltage when DNA adheres to the sensor surface. To do this, we place the structure in an LCR meter and measure the capacitance while we step the voltage. Figure 7A shows the general waveform for a typical C-V curve measured on our LCR meter. The DC voltage was stepped at 0.1 volts every second between -3 volts and 3 volts. The frequency was held at $20 \mathrm{~Hz}$ coupled with a $20 \mathrm{mV} \mathrm{AC}$ signal. The imposed AC signal was used to measure the capacitance. $20 \mathrm{~Hz}$ was chosen as it produced a clear $\mathrm{CV}$ curve with large capacitance values. Frequencies above $1 \mathrm{kHz}$ gave unstable results and inconsistent readings.

The graph also shows a low frequency $\mathrm{C}-\mathrm{V}$ curve in solutions of varying $\mathrm{pH}$. The shift expressed by different $\mathrm{pH}$ levels are represented by a shift in the flatband voltage. This is caused by a chemical reaction happening on the surface resulting in ion exchange. The shift seen in the graph is approximately 0.5 volts between a $\mathrm{pH}$ level of 6.8 and 12.3. This corresponds to a maximal change of $15 \mathrm{nF}$ when the optimal bias point of $-1.1 \mathrm{~V}$ is chosen (chosen from the left peak in the differential capacitance graph). Buffered solutions of known $\mathrm{pH}$ were used here so the effects of a wet reference electrode do not interfere with the test solution. It was noticed that Silicon dioxide is an interesting material that exhibits acidic properties. When placed in a basic solution, the acidic properties of $\mathrm{SiO}_{2}$ react with the basic electrolyte to produce salt and water [14]. The chemical reaction is:

$$
\mathrm{SiO}_{2}+2 \mathrm{NaOH} \rightarrow \mathrm{Na}_{2} \mathrm{SiO}_{3}+\mathrm{H}_{2} \mathrm{O}
$$

This is why a noticeable shift in flatband voltage can be observed in figure 7A for a $\mathrm{pH}$ of 12.3. This is how a pH field effect sensor works. Silicon dioxide is probably not the best material for a $\mathrm{pH}$ sensor since it only reacts to basic solutions. There are far better amphoteric materials out there which respond to both basic and acidic solutions. From figure 7A it is possible to calculate the optimal bias point by differentiating the $\mathrm{C}-\mathrm{V}$ curve. Figure 7B shows the result after the calculation. The peaks show the regions where the slope is steepest. A larger peak corresponds to a steeper curve - the most sensitive region for measurement. The $\mathrm{C}-\mathrm{V}$ curve closely resembles the characteristic curve of a MOS capacitor. The principle configuration is basically the same, except the gate in an EIS setup is the reference electrode in a solution rather than a metal/polysilicon contact. 


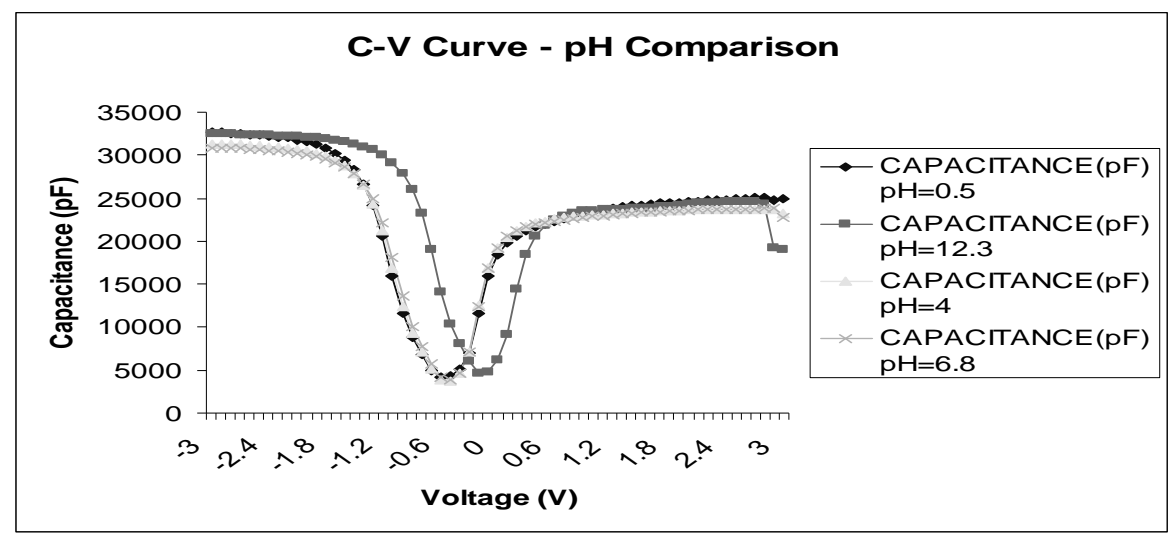

Figure 7A

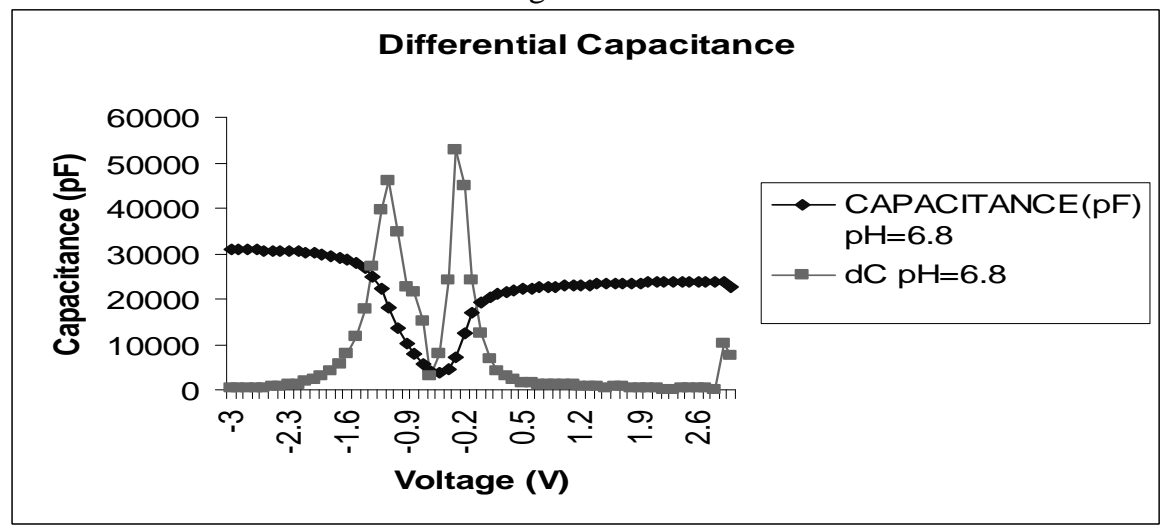

Figure 7B

Figure 7. A:CV Curve using various electrolytes of different $\mathrm{pH}$

B: Differential Capacitance for slope detection

\subsection{Time Drift}

An important factor to take into account in all the experiments was the time drift factor. Time drift was tested by allowing the experimental setup (from figure 5) to sit undisturbed over a length of time, while taking measurements at regular intervals. Our experiments show that a drift in the CV plot was significant in the first 30 minutes. After 30minutes, the flatband voltage has shifted 3volts and the accumulation capacitance had dropped over $5 \mathrm{nF}$. From figure 8 we have come to the conclusion that dissolved potassium ions (which are positively charged) have a preference for the surface of the oxide.

The positive ions are thought to be attracted by the negative carriers in our n-type substrate. $\mathrm{KCl}$ was chosen because it only had 2 elements which dissolved in water $\left(\mathrm{K}^{+}\right.$and $\left.\mathrm{Cl}_{2}{ }^{-}\right)$. Having only 2 elements means we could predict which element was causing the $\mathrm{CV}$ curve to be displaced due to its charge. In this case, we could easily deduce that potassium ions were causing the shift because it is shifting more towards the negative region of the curve. The reduction of capacitance in the accumulation regime is a result of the congregation of potassium ions near the surface. After a certain time, the system reaches equilibrium where the shift gets smaller and smaller until it is no longer displaced. During this state, the ions are thought to have reached maximum compactness (due to repulsion of like positive charges) near the surface of the oxide. Any added ions beyond this state cannot be "sensed" because it is too far away from the substrate. Therefore, before all measurements are to be made, the system needs to equilibrate for about 30 minutes. This needs to be done each time a new substrate is used. 


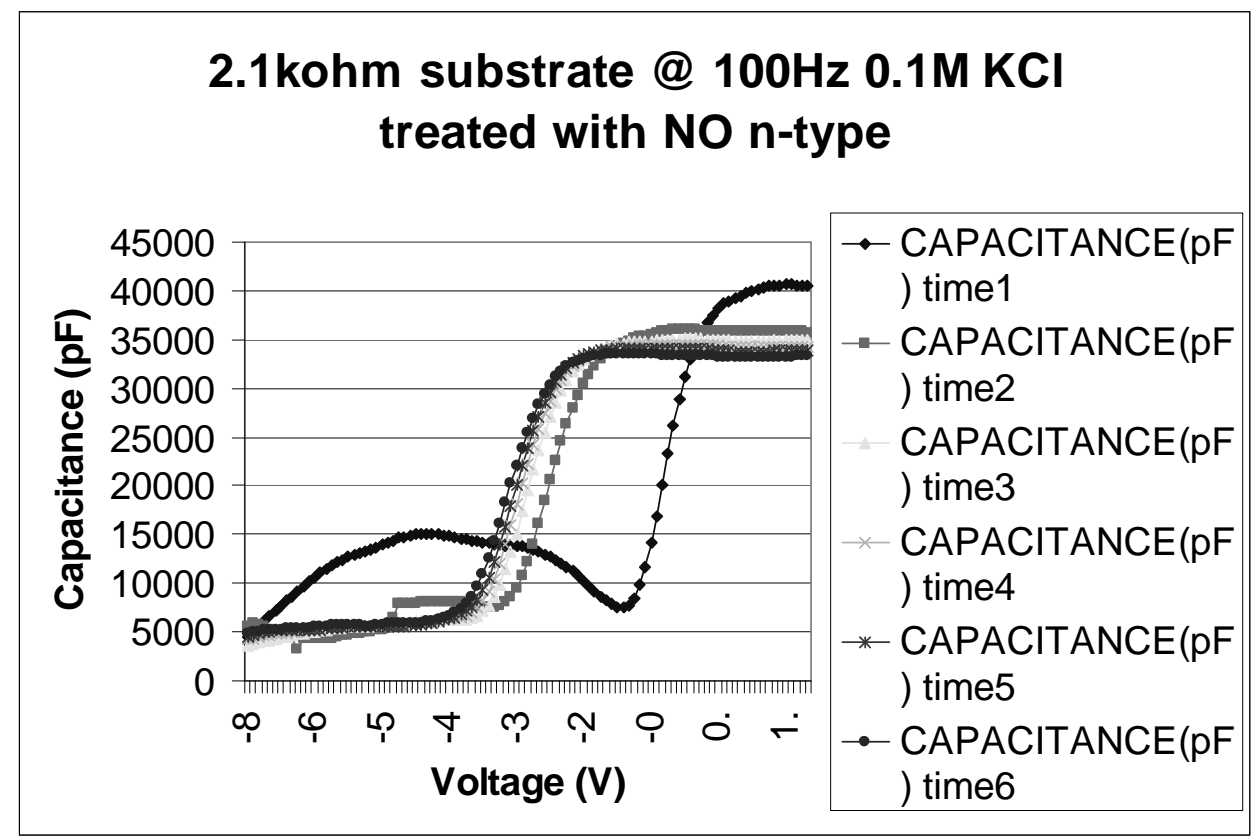

Figure 8. $\mathrm{CV}$ plot at difference time intervals in a $\mathrm{KCl}$ solution. Each plot was taken at 5 minute intervals.

\subsection{Frequency Sweep}

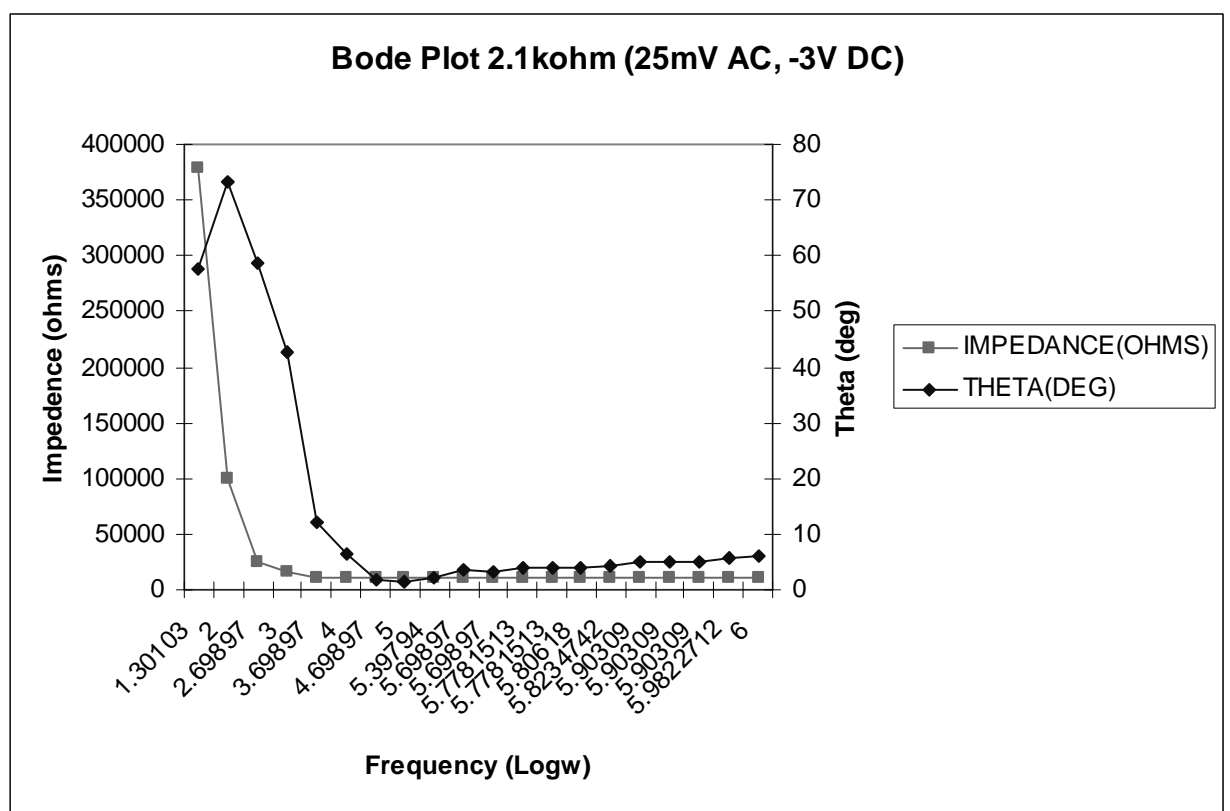

Figure 9. A bode plot showing the impedance and phase shift at frequencies ranging from $20 \mathrm{~Hz}$ to $1 \mathrm{Mhz}$

It is necessary to find which frequencies are best suited for monitoring DNA attachment and hybridisation. Figure 9 shows that for this particular substrate, a frequency anywhere between $20 \mathrm{~Hz}$ to $10 \mathrm{kHz}$ will give a capacitive response. $100 \mathrm{~Hz}$ seems to give peak capacitive response and model an almost ideal capacitor. The double layer capacitance can also be calculated by tracing the impedance intercept all the way past $\log \omega=0$ when biased in accumulation. In our case, the depletion capacitance will dominate over the double layer capacitance so this will not be necessary. We have chosen to perform our measurements at $100 \mathrm{~Hz}$. 


\section{FUTURE WORK}

We hope to microfabricate the system onto silicon and integrate it into a CMOS compatible process so that it is portable and cheap.

So far we've only discussed the EIS structure to be used as a single sensor platform. This has many disadvantages such as drift, ion interference, noise, reference electrode drifts, no ability to compare DNA mismatches, and measures only one species at a time.

We aim to improve the sensor by introducing differential sensing. In differential sensing two sensors are required to measure 1 species. One sensor will act as a reference whilst the other will be the active or working sensor. The active sensor is functionalized with capture probe A and second sensor with capture probe B. A solution containing a sequence complimentary to capture probe A is injected and the net capacitance measured. The net capacitance is the result of the difference in capacitance between the two sensors. This approach helps reduce noise caused by counter-ion interference, non specific binding and the "floating" electrode problem.

\section{CONCLUSION}

So far it was proved that it detects changes in $\mathrm{pH}$ and can be used as a simple $\mathrm{pH}$ sensor. An EIS structure has been highlighted here as the platform for pathogen detection using the field effect phenomenon. The structure is improved upon by eliminating noise and interference using a pair of sensors. The results highlighted in the paper show the single sensor system is moving toward becoming a biological sensor. 


\section{REFERENCES}

[5] S.MiddelHoek, S.A. Audet, Silicon Sensors, Academic Press, 1989

[6] W.L.W.Hao, D.Trau, N.Sucher, M.Wong, Y.Zohar, Micropatterns on demand using surface chemistry technology, (2002)

[7] A.S.Grove, Physics and Technology of Semiconductor Devices, Wiley, New York, (1967)

[8] E.Cooper, J.Fritz, G.Wiegand, P.Wagner, S.Manalis, Robust Microfabricated Field Effect Sensor for Monitoring Field Effect Sensor For Monitoring Molecular Absorption in Liquids, (2001)

[9] D.Woermann, Cellular and Molecular Biology Letters (Volume 6), 2001, pp53-57 Germany

[14] R.Chang, Chemistry $5^{\text {th }}$ Edition, McGraw Hill, 1994, USA Chpt:8.6 pg323. 